\title{
Erratum to: Preventive effects of COX-2 inhibitor, celecoxib on renal tubular injury induced by shock wave lithotriptor
}

\author{
Hyoung Keun Park $\cdot$ Hae Won Lee \\ Kwang Soo Lee $\cdot$ Jong Sun Choi $\cdot$ \\ Byong Chang Jeong $\cdot$ Hyeon Hoe Kim
}

Published online: 2 April 2010

(C) Springer-Verlag 2010

Erratum to: Urol Res

DOI 10.1007/s00240-009-0243-z

The affiliation of Park Hyoung Keun has changed recently to Department of Urology, College of Medicine, Konkuk University, Chungju, Korea.

The online version of the original article can be found under doi:10.1007/s00240-009-0243-z.

H. K. Park

Department of Urology, College of Medicine,

Konkuk University, Chungju, Korea

H. W. Lee - K. S. Lee

Department of Urology, College of Medicine,

Dongguk University, Gyeongju, Korea

\section{J. S. Choi}

Department of Pathology, College of Medicine,

Dongguk University, Gyeongju, Korea

B. C. Jeong

Department of Urology, College of Medicine,

Sungkyunkwan University, Seoul, Korea

H. H. Kim ( ()

Department of Urology, College of Medicine,

Seoul National University, Seoul, Korea

e-mail: hhkim@snu.ac.kr 